\title{
A Novel Thermal Infrared Enthalpimetric Method for Fast, High-Throughput Determination of the Content Uniformity of Captopril Tablets
}

\author{
Flavia M. Dalla Nora, ${ }^{a}$ Alessandra S. Oliveira, ${ }^{a}$ Bruna N. Lucas, ${ }^{a}$ Daniele F. Ferreira, ${ }^{a}$ \\ Fabio A. Duarte, ${ }^{b}$ Adilson B. Costa, ${ }^{c}$ Fabiana E. B. Silva ${ }^{d}$ and Juliano S. Barin ${ }^{\circledR} *, a, d$
}

\author{
${ }^{a}$ Departamento de Tecnologia e Ciência dos Alimentos, \\ Universidade Federal de Santa Maria, 97105-900 Santa Maria-RS, Brazil \\ ${ }^{b}$ Departamento de Química, Universidade Federal de Santa Maria, \\ 97105-900 Santa Maria-RS, Brazil \\ 'Programa de Pós-Graduação em Sistemas e Processos Industriais, \\ Universidade de Santa Cruz do Sul, 96815-900 Santa Cruz do Sul-RS, Brazil \\ 'Programa de Pós-Graduação em Ciências Farmacêuticas, \\ Universidade Federal do Pampa (UNIPAMPA), 97501-970 Uruguaiana-RS, Brazil
}

\begin{abstract}
Content uniformity $(\mathrm{CU})$ is a parameter used to ensure the uniformity of the amount of active substances in pharmaceutical preparations. It is often evaluated in the quality control of pharmaceutical dosage forms, and a novel method was proposed for $\mathrm{CU}$ determination using thermal infrared enthalpimetry (TIE). Captopril tablets were dissolved in 24-well microplates and the heat released from the reaction of captopril and $\mathrm{I}_{2}$ was monitored with an infrared camera. Some experimental parameters were adjusted, as captopril/ $/ \mathrm{I}_{2}$ ratio, stirring speed and dispensing rate, and the most suitable conditions were 1.00:1.00 (v/v), $250 \mathrm{rpm}$ and $0.57 \mathrm{~mL} \mathrm{~s}^{-1}$, respectively. Interferences from tablet adjuvants (e.g., starch, cellulose and lactose) were not observed. The results obtained by TIE did not differ statistically (Student's $t$-test, $p>0.05$ ) from those from official method (UV spectrophotometry) described in pharmacopeia. The TIE method required 31 and 12 times less reagent and time, respectively. Therefore, the proposed method was suitable for routine analysis and agrees with the requirements of green analytical chemistry.
\end{abstract}

Keywords: active pharmaceutical ingredients, enthalpimetric analysis, content uniformity determination, green analytical chemistry, thermography

\section{Introduction}

Quality control is essential in the pharmaceutical industry to assure the quality and safety of the active pharmaceutical ingredients presented in different formulations. Captopril (1-[(2S)-2-methyl-3-sulfanylpropanoyl]- $L$-proline) is an active pharmaceutical ingredient largely used for antihypertension due to its inhibitor effect on the angiotensin-converting enzyme. ${ }^{1}$ Quality control of captopril is performed according to the methods described in pharmacopeias, including the evaluation of content uniformity (CU).

The $\mathrm{CU}$ is used as a parameter to assure the uniformity in the amount of drug present among dosage units, as

*e-mail: juliano@ufsm.br solutions, powders, granules, sterile solids, tablets and hard capsules. Weight variation could be used instead $\mathrm{CU}$ in some cases. The choice of the method to be used considers the pharmaceutical form, amount and proportion of the drug in the dosage form. In this way, according to the monograph of captopril in Brazilian Pharmacopoeia, the CU evaluation is recommended for tablets. ${ }^{2}$ For this purpose, 10 solid-dosage forms should be diluted in ethanolic solution with further spectrophotometric measurement at $212 \mathrm{~nm}$ for further calculation of acceptance value (AV). ${ }^{2}$ Other solvents can be used to dissolve the tablets, such as $0.1 \mathrm{~mol} \mathrm{~L}^{-1} \mathrm{HCl}$ for further spectrophotometric determination of captopril. ${ }^{3}$ In addition, $\mathrm{CU}$ can also be determined using high-performance liquid chromatography (HPLC), a method performed using reference solutions and samples, both diluted in the mobile phase and submitted to 
sonication for $5 \mathrm{~min} .{ }^{4,5}$ Despite the widespread use of UV spectrophotometry and HPLC for CU evaluation, some drawbacks, such as the time required and limited sample throughput, have impaired their use in routine.

Recently, thermal infrared enthalpimetry (TIE) was proposed as a new technique that uses an infrared camera to monitor differences in the temperature of solutions for neutralization, precipitation, complexation and redox reactions. This technique allows the simultaneous evaluation of several microplate wells, to which reagents are added by a multichannel pipette. The heat released from reactions is measured and the difference of temperature before and after reaction is determined, which is proportional to the amount of analyte. This approach was used to determine calcium carbonate and ferrous sulfate in tablets and sodium chloride in physiologic sterile saline solution. ${ }^{6}$ The agreement of TIE with the conventional method ranged from 96 to $101 \%$, showing good accuracy and increased sample throughput in comparison to the conventional titration. Other applications in this field include the determination of vinegar acidity, ${ }^{7}$ content and acidity of pickled vegetable brine, ${ }^{8}$ alcohol content of distilled beverages and wines ${ }^{9}$ and saponification value in edible oils. ${ }^{10}$ These studies achieved an increase in throughput and decrease in time, reagent consumption and energy requirements.

Therefore, TIE is proposed as a simple and rapid method to determine CU in captopril tablets. Sample preparation and analysis were performed in the same reactor, avoiding excessive sample handling. The effect of adjuvants (starch, cellulose and lactose) used in the captopril formulations and the influence of reagents ratio, stirring speed, and solution dispensing rate were evaluated in order to obtain the lowest variation among measurements. The results were compared with those from the conventional method described in pharmacopeias.

\section{Experimental}

\section{Samples and chemicals}

Samples of captopril tablets $(50 \mathrm{mg})$ from four different manufacturers (named 1, 2, 3 and 4) were purchased from a local market (Santa Maria, RS, Brazil). Sample 1 was used to evaluate the analytical parameters. Hydroalcoholic solutions $(1: 1, \mathrm{v} / \mathrm{v})$ were prepared using absolute ethanol (99.8\%, Dinâmica, Brazil) and ultrapure water was obtained from the MilliQ system (Direct-Q $3 \mathrm{UV}, 18.2 \mathrm{M} \Omega \mathrm{cm}$, Millipore Corp., USA). A reference solution of $0.25 \mathrm{~mol} \mathrm{~L}^{-1}$ iodine was prepared in $30 \%(\mathrm{~m} / \mathrm{v})$ potassium iodide $(99.0 \%$, Vetec, Brazil) hydroalcoholic solution using solid iodine $(99.9 \%$, Neon, Brazil). This solution was used as a reagent to stoichiometric excess in the TIE method. In order to determine the CU by TIE, a reference solution containing $50 \mathrm{mg}$ of captopril in $1.2 \mathrm{~mL}$ was prepared using captopril with $99.8 \%$ of purity.

Instrumentation

Reactions were carried out in a disposable, polystyrene, 24-well microplate (Nest Biotechnology, China), with each well with total volume of $3.0 \mathrm{~mL}$. All reagents were introduced into the reactors using an electronic multichannel pipette (Research Pro 1200, Eppendorf, Germany) with eight channels and volume ranging from 0.050 to $1.200 \mathrm{~mL}$. A magnetic stirrer (Centauro, Brazil) was used for stirring. A long-wave infrared camera (7.5-13.0 $\mu \mathrm{m}$, $320 \times 240$ pixels, $30 \mathrm{~Hz}$, FLIR E60 model, FLIR, USA) was used to simultaneously determine the temperature in the wells. Images were processed using ResearchIR software (FLIR, version 3.5). An ultrasonic bath $(25 \mathrm{kHz}$, model D-78224, Elmasonic, Germany) was used to dissolve the tablets and a UV-Vis spectrophotometer (model 8453, Agilent, USA) was used at $212 \mathrm{~nm}$ to determine the $\mathrm{CU}$ of captopril tablets according to the Brazilian Pharmacopoeia method. ${ }^{2}$ The energy consumed was obtained using a power meter (Fluxe 43B model, Fluke Corporation, Everett, USA). For TIE, the energy consumption of the notebook, infrared camera, magnetic stirrer, as well as the power required to charge the multichannel electronic pipette was measured. For pharmacopeial method the energy consumed by the spectrophotometer was considered during the determination of CU.

\section{Optimization of parameters for TIE analysis}

The total amount of adjuvants used in the evaluated captopril formulations was around $150 \mathrm{mg} .{ }^{11}$ Given the main adjuvants used in such formulations, the effects of starch, cellulose and lactose were evaluated. This evaluation was performed by using 50,100 and $150 \mathrm{mg}$ of each adjuvant or a mix containing $50 \mathrm{mg}$ of each were added to all wells. After, $1.2 \mathrm{~mL}$ of solution containing $50 \mathrm{mg}$ of captopril was then added. The difference in temperature $(\Delta \mathrm{T})$ values obtained were compared to a reference solution $(1.2 \mathrm{~mL})$ containing $50 \mathrm{mg}$ of captopril.

The ratio between captopril and iodine solution (from 0.50:1.00 to 1.50:1.00), stirring speed (from 100 to $300 \mathrm{rpm}$ ), and dispensing rate (from 0.46 to $0.71 \mathrm{~mL} \mathrm{~s}^{-1}$ ) were evaluated. For this purpose, a mix containing starch, cellulose and lactose $(150 \mathrm{mg})$ was previously added to each well. To these reactors, a reference solution $(1.2 \mathrm{~mL})$ was added, followed by $1.2 \mathrm{~mL}$ of $0.25 \mathrm{~mol} \mathrm{~L}^{-1} \mathrm{I}_{2}$ solution 
as a reagent in stoichiometric excess. All parameters were compared using $\Delta \mathrm{T}$ values.

The time required to disintegrate and dissolve tablets was then evaluated. Tablets were inserted into each well, followed by $1.2 \mathrm{~mL}$ of hydroalcoholic solution (1:1). The temperature variation was monitored under the addition of $0.25 \mathrm{~mol} \mathrm{~L}^{-1} \mathrm{I}_{2}$ solution $(1.2 \mathrm{~mL})$ until the temperature remained constant. All results were evaluated using the relative standard deviation $(\mathrm{RSD})$ of measurements $(\mathrm{n}=10)$ and by comparing results with those obtained with captopril reference solution $(n=10)$.

\section{Determination of CU by TIE}

Tablets were inserted into each well using tweezers, and $1.2 \mathrm{~mL}$ of hydroalcoholic solution (1:1) was added. Wells were maintained under stirring for 16 min using a polytetrafluoroethylene-covered magnetic stir bar $(3.0 \times 6.5 \mathrm{~mm})$. After dissolution, stoichiometric excess of $\mathrm{I}_{2}$ solution $(0.25 \mathrm{~mol} \mathrm{~L}-1,1.2 \mathrm{~mL})$ was added, as shown in Figure 1. The temperature was monitored by an infrared camera fixed at a distance of $40 \mathrm{~cm}$ from the microplates, and a circle covering $46.8 \%$ of the total well area was used to monitor the reactions, according to previous work. ${ }^{9}$ The average of the temperature data was plotted to form an enthalpogram. Temperature variation was calculated using the equation $\Delta \mathrm{T}=\mathrm{T}_{\mathrm{f}}-\mathrm{T}_{\mathrm{i}}$, where $T_{f}$ is the final temperature after the reaction and $T_{i}$ is the initial temperature before the reaction. The $T_{f}$ and $T_{i}$ values were obtained by averaging the values from $2 \mathrm{~s}$ before and after the addition of reagent in stoichiometric excess. ${ }^{6}$ For all measurements, a video was recorded covering all steps before and after reaction. The relatively humidity and temperature were corrected in the software.

In the same way, reference solution containing the same amount of captopril (50 mg) was evaluated. The amount of captopril was determined based on temperature variation, using equation 1 :

Captopril $(\mathrm{mg})=50 \mathrm{mg} \times \frac{\Delta \mathrm{T}_{1}}{\Delta \mathrm{T}_{2}}$

where $\Delta T_{1}$ is the difference in temperature for the sample solution $\left({ }^{\circ} \mathrm{C}\right)$ and $\Delta \mathrm{T}_{2}$ is the difference in temperature for the reference solution $\left({ }^{\circ} \mathrm{C}\right)$. Afterwards, the $\mathrm{CU}$ and $\mathrm{AV}$ were calculated based on the Brazilian Pharmacopoeia criteria. $^{2}$

\section{Determination of CU according to Brazilian Pharmacopoeia}

Ten tablets containing $50 \mathrm{mg}$ captopril were individually transferred to $50 \mathrm{~mL}$ volumetric flasks containing $5 \mathrm{~mL}$ of water. $25 \mathrm{~mL}$ of a hydroalcoholic solution (1:1) were added to each flask, which were sonicated for $15 \mathrm{~min}$ in the ultrasonic bath and mechanically agitated for $15 \mathrm{~min}$. After tablets dissolution, the content was filtered, and an aliquot of $0.5 \mathrm{~mL}$ was transferred to a $25 \mathrm{~mL}$ volumetric flask and completed with the same hydroalcoholic solution. The same procedure was performed for a reference solution containing $50 \mathrm{mg}$ of captopril and blank solution. Blanks, samples and reference solutions were evaluated by UV-Vis spectrophotometer at $212 \mathrm{~nm}$ and the CU was determined. Then, the acceptance value was calculated according to the Brazilian Pharmacopoeia criteria.

\section{Results and Discussion}

Influence of adjuvants used in captopril tablets on TIE measurements

Determination of CU by TIE is based on the reaction between captopril and iodine (Figure 2). After oxidation

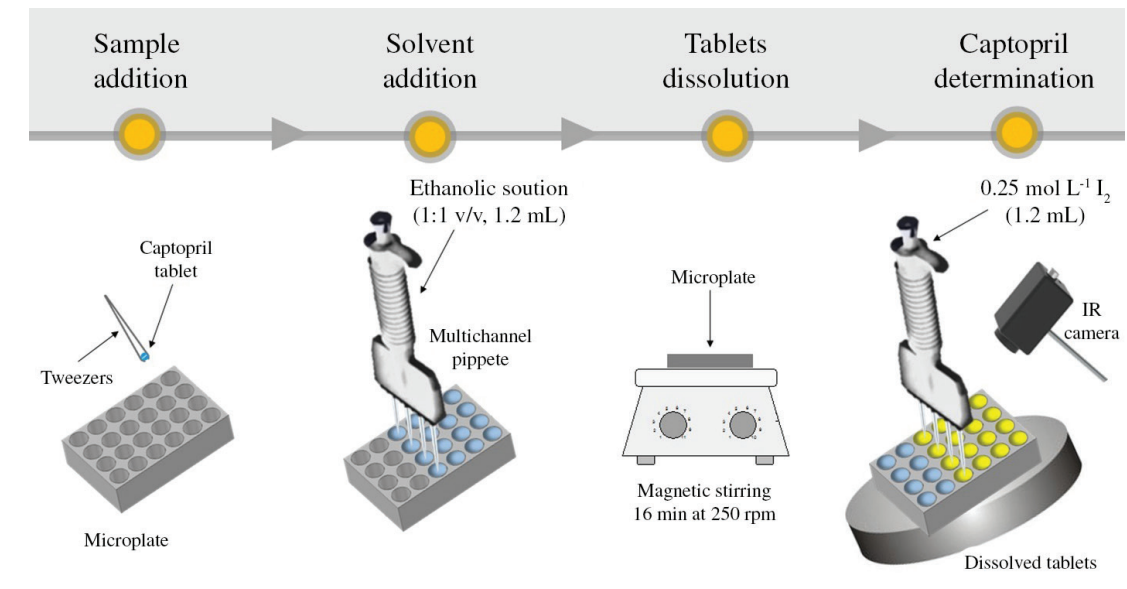

Figure 1. Procedure used to determine captopril content in tablets by TIE method. 
by $\mathrm{I}_{2}$, captopril becomes captopril disulfide, the main impurity present in raw captopril material. ${ }^{12,13}$ Because the TIE method is based on this reaction, this impurity does not interfere, because only captopril and not the captopril disulfide reacts with iodine.

Prior to optimizing the parameters of the TIE measurements, a study regarding the main adjuvants used in captopril tablets was performed to evaluate the possible interference of the matrix during reactions. According to the Brazilian National Health Surveillance Agency (ANVISA) database, ${ }^{11}$ the main adjuvants used in captopril tablets are monohydrate lactose, microcrystalline cellulose and pregelatinized starch. The average weight of the tablets was around $200 \mathrm{mg}$. Since the captopril samples weighed $50 \mathrm{mg}$, the influence of $150 \mathrm{mg}$ of different adjuvants was evaluated: 50, 100 and $150 \mathrm{mg}$ of each and a mix with $50 \mathrm{mg}$ of each adjuvant (Figure 3).

According to the data (Figure 3 ), the $\Delta \mathrm{T}$ of all reactions ranged from 1.01 to $1.04^{\circ} \mathrm{C}$, with no significant differences (Tukey's test, $p>0.05$ ), showing no effect of the matrix in determining captopril by the proposed method. All subsequent optimizations were thus carried out using $150 \mathrm{mg}$ of starch.

\section{Optimization of TIE parameters for analysis}

Prior to captopril determination by TIE, optimization was performed to identify suitable conditions. First, the captopril/ $\mathrm{I}_{2}$ ratio was evaluated with a total volume of $2.4 \mathrm{~mL}$. The highest temperature variation in the reactions was achieved with a ratio of 1.00:1.00 (v/v; Figure 4a), which could be associated with better homogenization. Using ratios other than 1.00:1.00 lead to lower temperature variation with increase of the deviations among measurements.

Since the solutions were homogenized using a magnetic stir bar, it is important to evaluate the stirring speed. For this optimization, captopril/ $\mathrm{I}_{2}$ solution ratio of 1.00:1.00 (v/v) was considered. As Figure $4 \mathrm{~b}$ shows, there were no significant differences (Tukey's test, $p>0.05$ ) in the average, but low stirring speeds increased RSD values, probably as a consequence of inefficient homogenization. The most suitable results were achieved at $250 \mathrm{rpm}$ stirring speed.

The $\mathrm{I}_{2}$ dispensing rate was also evaluated. According to the results (Figure 4c), significant differences were identified only for 0.50 and $0.57 \mathrm{~mL} \mathrm{~s}^{-1}$ (Tukey's test, $p>$ 0.05). Considering the RSD values, the higher dispensing
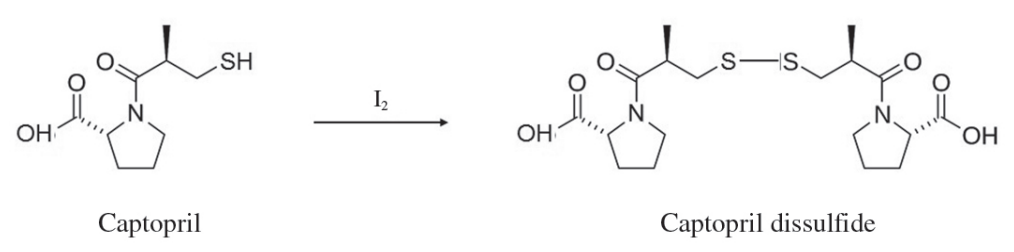

Figure 2. Reaction between captopril and iodine used in the proposed TIE method.

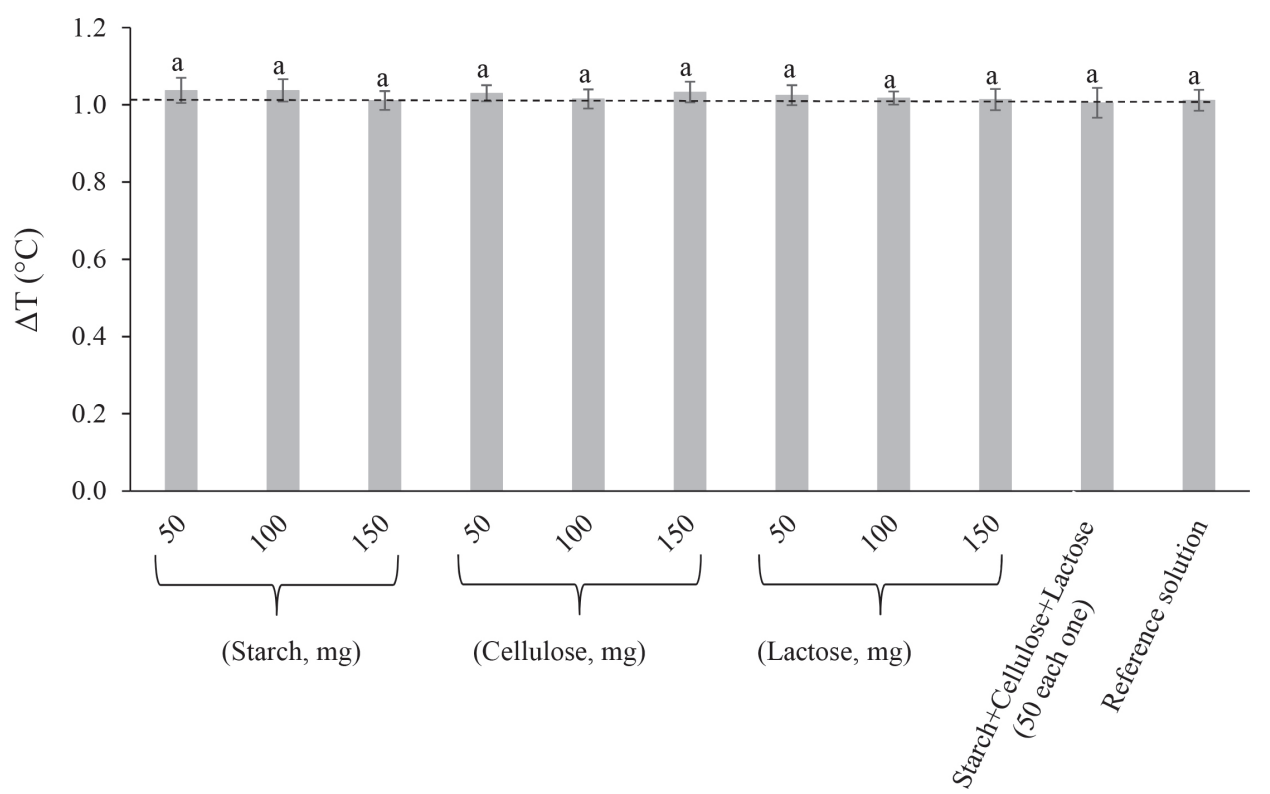

Figure 3. Effect of main adjuvants used in captopril tablets on TIE measurements $(\mathrm{n}=10)$, with captopril amount of $50 \mathrm{mg}$ in each well. Dashed line indicates the temperature of reference solution without adjuvants. Same letters indicate no significant difference (Tukey's test, $p>0.05$ ). 


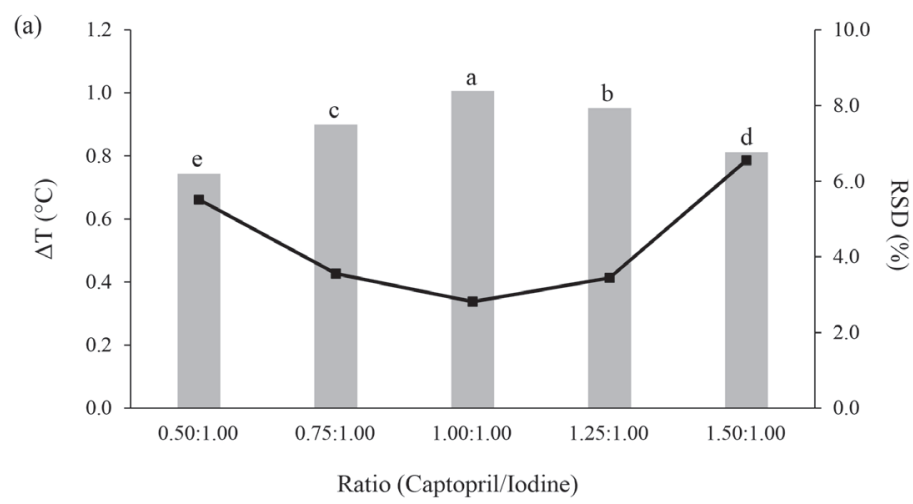

Fixed conditions

Stirring speed: $200 \mathrm{rpm}$ Dispensing rate: $0.57 \mathrm{~mL} \mathrm{~s}^{-1}$

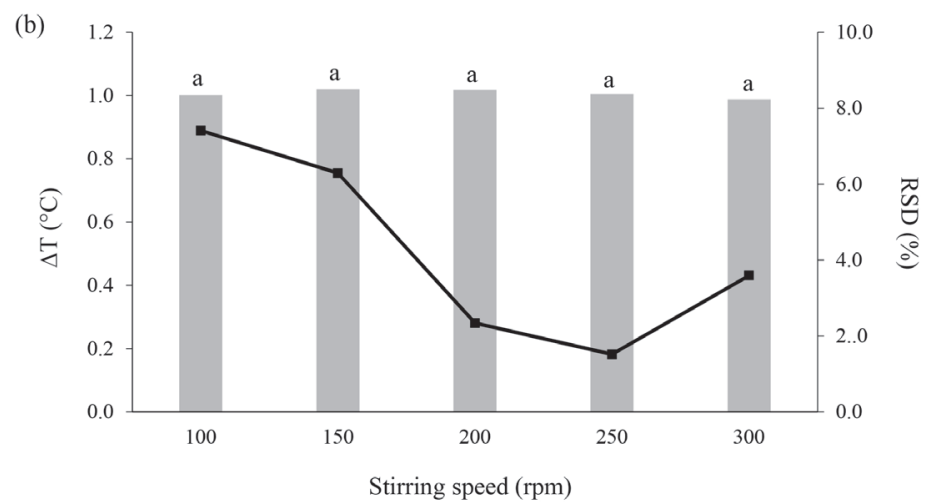

Fixed conditions

Ratio: 1.00:1.00 Dispensing rate: $0.57 \mathrm{~mL} \mathrm{~s}^{-1}$

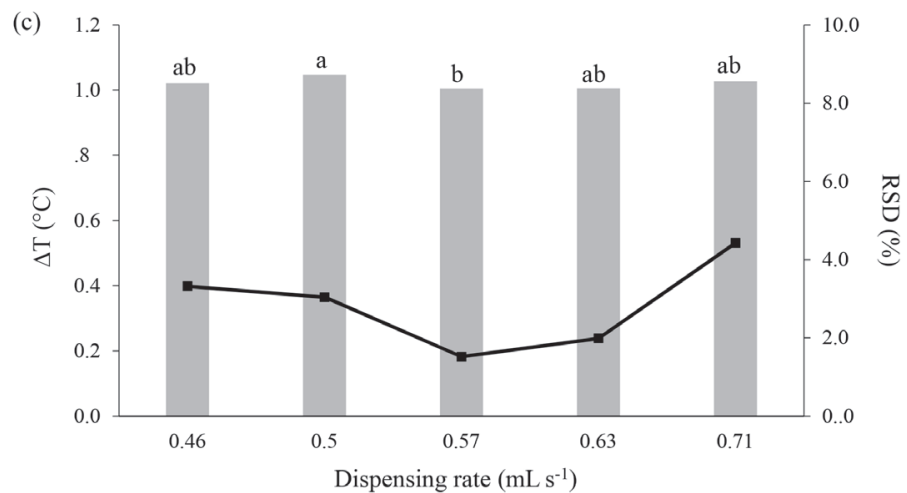

Fixed conditions

Ratio: 1.00:1.00 Stirring speed: $250 \mathrm{rpm}$

Figure 4. Influence of TIE parameters: (a) captopril/iodine solutions ratio; (b) stirring speed and (c) dispensing rate. Same letters indicate no significant difference (Tukey's test, $p>0.05$ ).

rate provided the highest variation in results, probably as a consequence of the loss of solution from wells. High variation among the measurements was also observed for lower dispensing rates $\left(0.46\right.$ and $\left.0.50 \mathrm{~mL} \mathrm{~s}^{-1}\right)$. This could be explained by the time that pipette tips remained in front of the camera, which impaired the measurement of temperature variation and consequently the deviation of measurements. A dispensing rate of $0.57 \mathrm{~mL} \mathrm{~s}^{-1}$ was chosen, taking into account the lower deviations and the average $\left(1.00 \pm 0.02{ }^{\circ} \mathrm{C}\right)$, which was very close to the reference solution without adjuvants $\left(1.01 \pm 0.03{ }^{\circ} \mathrm{C}\right)$. Therefore, the parameters chosen for the determination of captopril were a captopril// $\mathrm{I}_{2}$ ratio of 1.00:1.00 (v/v), stirring speed of $250 \mathrm{rpm}$ and dispensing rate of $0.57 \mathrm{~mL} \mathrm{~s}^{-1}$.

\section{Determination of captopril in tablets}

Compression during the fabrication of captopril tablets could be different, therefore, disintegration and dissolution are required prior to the determination to ensure total drug dissolution. In this regard, $1.2 \mathrm{~mL}$ of hydroalcoholic solution was used to dissolve each tablet. Figure 5 shows the dissolution and disintegration profile. The tablets from manufacturers 1 and 3 dissolved in 4 min, while samples obtained from manufacturers 2 and 4 required more time for total dissolution, dissolving in 10 and $12 \mathrm{~min}$, respectively. This distinct behavior could be explained by the different procedures and proportions of adjuvants that each manufacturer used in the tablets, which affects 
the compression and consequently the disintegration and drug dissolution.

Considering these differences in dissolution time, all samples underwent $16 \mathrm{~min}$ of stirring. Captopril content was determined in the four commercial samples by the TIE method and compared with the results obtained by the conventional method. ${ }^{2}$ As shown in Table 1, the proposed method was accurate, with no significant differences (Student's $t$-test, $p>0.05$ ) for all samples evaluated between both methods. Regarding the precision, lower values of RSD were achieved with the proposed method than with conventional determination, with influence on the AV (Table 1).

The requirements for dosage uniformity (according to Brazilian Pharmacopeia) ${ }^{2}$ were reached for all evaluated samples. Acceptance values were calculated for all samples using both methods; for 10 units, their $\mathrm{AVs}$ were lower than 15.0, ensuring the consistency of dosage units without the

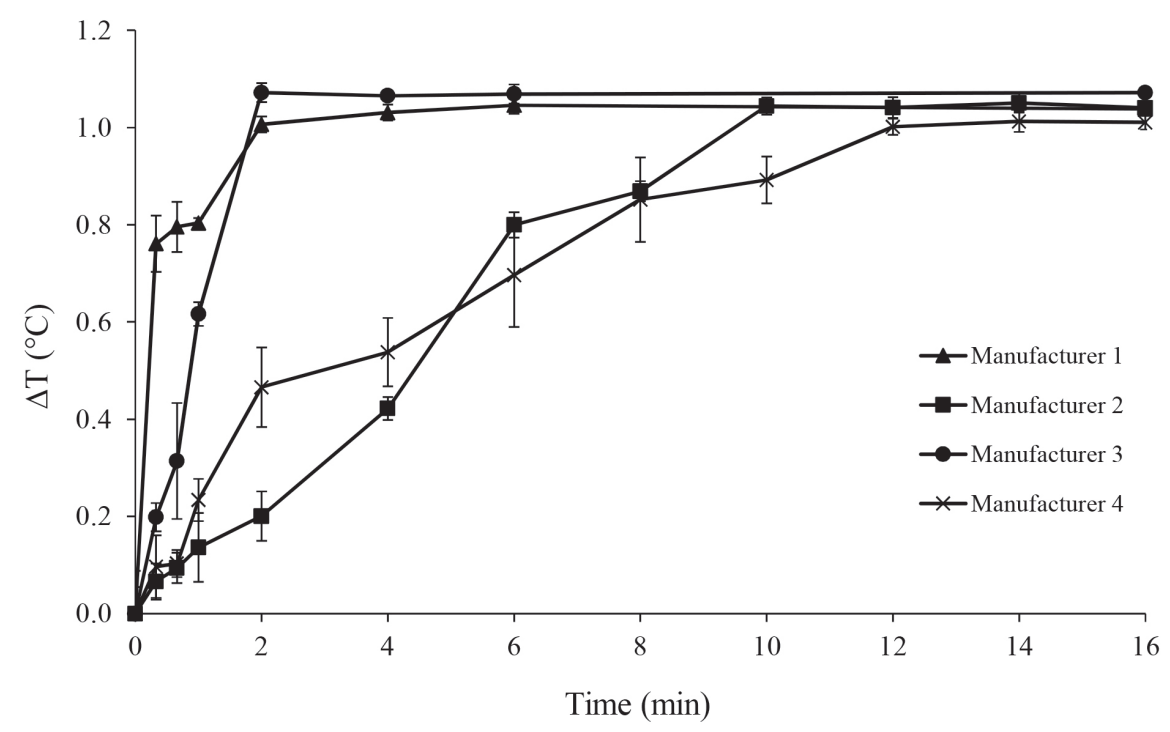

Figure 5. Profile of tablets dissolution from different manufacturers $(n=4)$.

Table 1. Results for content uniformity of captopril $50 \mathrm{mg}$ tablets by TIE and conventional method described in Brazilian Pharmacopeia (Conv.). The acceptance value was calculated based on Brazilian Pharmacopeia parameters. ${ }^{2}$ No significant difference was observed between TIE and conventional method (Student's $t$-test, $p>0.05$ )

\begin{tabular}{|c|c|c|c|c|c|c|c|c|}
\hline \multirow{3}{*}{ Tablet } & \multicolumn{8}{|c|}{ Manufacturer } \\
\hline & \multicolumn{2}{|c|}{1} & \multicolumn{2}{|c|}{2} & \multicolumn{2}{|c|}{3} & \multicolumn{2}{|c|}{4} \\
\hline & TIE & Conv. & TIE & Conv. & TIE & Conv. & TIE & Conv. \\
\hline 1 & 51.3 & 50.5 & 50.5 & 50.5 & 54.4 & 53.6 & 49.9 & 49.9 \\
\hline 2 & 51.2 & 50.1 & 49.7 & 54.0 & 51.8 & 53.7 & 50.1 & 49.5 \\
\hline 3 & 50.6 & 50.6 & 51.4 & 49.8 & 52.3 & 54.6 & 50.5 & 49.4 \\
\hline 4 & 51.1 & 48.4 & 51.9 & 53.1 & 53.4 & 52.7 & 50.5 & 49.4 \\
\hline 5 & 49.7 & 50.2 & 52.0 & 53.0 & 51.3 & 52.0 & 49.6 & 49.7 \\
\hline 6 & 50.4 & 50.2 & 52.1 & 52.0 & 51.8 & 53.9 & 50.5 & 49.5 \\
\hline 7 & 51.6 & 51.0 & 51.0 & 52.0 & 52.9 & 52.5 & 51.0 & 50.3 \\
\hline 8 & 49.8 & 50.4 & 51.3 & 49.7 & 51.8 & 54.3 & 49.5 & 49.5 \\
\hline 9 & 51.9 & 51.9 & 51.1 & 53.4 & 52.4 & 52.7 & 50.6 & 50.4 \\
\hline 10 & 51.3 & 51.6 & 51.9 & 50.9 & 51.9 & 51.7 & 49.9 & 51.9 \\
\hline Mean & 50.9 & 50.7 & 51.3 & 51.9 & 52.4 & 53.2 & 50.2 & 49.9 \\
\hline SD & 0.7 & 0.8 & 0.8 & 1.5 & 0.9 & 1.0 & 0.5 & 0.8 \\
\hline $\mathrm{RSD} / \%$ & 1.5 & 1.6 & 1.5 & 2.9 & 1.8 & 1.9 & 1.0 & 1.6 \\
\hline Acceptance value & 3.8 & 3.8 & 4.8 & 9.5 & 7.2 & 9.7 & 2.3 & 3.8 \\
\hline
\end{tabular}

TIE: thermal infrared enthalpimetry; RSD: relative standard deviation. 
requirement to analyze 20 more units. Since the proposed method yielded lower deviations among measurements, the $\mathrm{AVs}$ were lower than those when using conventional determination. Therefore, the effect of uncertainties from analytical method in the AV is reduced using TIE, which is an important benefit for $\mathrm{CU}$ evaluation.

\section{Evaluation of environmental impacts}

Routine chemical analysis in the chemical and pharmaceutical industries has environmental impacts. In this regard, the development and use of alternative technologies or methods, which decrease the environmental impact, is a trend. Some parameters related to environmental aspects were evaluated in order to compare the proposed method to conventional captopril determination. ${ }^{14,15}$

The miniaturized, proposed method consumed 31 times less alcohol than conventional captopril determination. The conventional method required 12 times more energy than the TIE method. The proposed method does employ KI and $\mathrm{I}_{2}$ as reagents, which are drawbacks compared to conventional CU determination. However, in terms of throughput, the TIE method allowed the determination of at least 24 replicates (considering only one microplate) in less than one-half hour.

It is important to mention that the use of microplates allowed the integration of analytical processes in the same vessel, minimizing random and systematic errors and impacting the precision of measurements. Thus, captopril dissolution and determination steps in the proposed method were made in the same reactor, which provided suitable accuracy and greater precision in the results compared with the conventional method.

\section{Conclusions}

The proposed TIE method allows the determination of the $\mathrm{CU}$ of $50 \mathrm{mg}$ captopril tablets more quickly and more precisely than does the conventional pharmacopeial method. Results for CU were satisfactory, considering that all samples agreed with the requirements of the Brazilian Pharmacopoeia. Time, energy and reagent consumption were reduced by more than 24,11 and 31 times, respectively, in the proposed method, which could therefore be considered more environmentally friendly than the conventional method. In addition, the integration of processes allows measurements with lower deviations, resulting in lower acceptance values compared with those in the Brazilian Pharmacopoeia. In addition, advantages of the proposed TIE method, such as the reduction of steps for sample preparation and higher throughput, make it suitable for routine analysis.

\section{Acknowledgments}

This study was financed in part by the Coordenação de Aperfeiçoamento de Pessoal de Nível Superior (CAPES, Brazil, Finance Code 001). The authors also thank the Conselho Nacional de Desenvolvimento Científico e Tecnológico (CNPq, Brazil) and the Fundação de Amparo à Pesquisa do Estado do Rio Grande do Sul (FAPERGS) for supporting this study.

\section{References}

1. Katzung, B. G.; Masters, S. B.; Trevor, A. J.; Basic \& Clinical Pharmacology, vol. 1, 12 $2^{\text {th }}$ ed.; McGraw-Hill Medical: New York, USA, 2012.

2. Brazilian Pharmacopeia, $5^{\text {th }}$ ed.; National Sanitary Surveillance Agency (ANVISA): Brasília, Brazil, 2010.

3. The Indian Pharmacopoeia; Government of India, Ministry of Health and Family Welfare: New Delhi, India, 1996.

4. The United States Pharmacopeia, USP 38; The United States Pharmacopeia Convention: Rockville, 2014.

5. Moreira, F. L.; Silva, E. O.; Bonfilio, R.; Santos, O. M. M.; Araujo, M. B.; Curr. Pharm. Anal. 2013, 9, 139.

6. Barin, J. S.; Tischer, B.; Oliveira, A. S.; Wagner, R.; Costa, A. B.; Flores, E. M. M.; Anal. Chem. 2015, 87, 12065.

7. Tischer, B.; Oliveira, A. S.; Ferreira, D. F.; Menezes, C. R.; Duarte, F. A.; Wagner, R.; Barin, J. S.; Food Chem. 2017, 215, 17.

8. Tischer, B.; Oliveira, A. S.; Costa, A. B.; Cichoski, A. J.; Barcia, M. T.; Wagner, R.; Barin, J. S.; J. Food Compos. Anal. 2017, 63,34 .

9. Oliveira, A. S.; Dalla Nora, F. M.; Mello, R. O.; Mello, P. A.; Tischer, B.; Costa, A. B.; Barin, J. S.; Talanta 2017, 171, 335.

10. Dalla Nora, F. M.; Oliveira, A. S.; Lucas, B. N.; Ferreira, D. F.; Duarte, F. A.; Mello, R. O.; Cichoski, A. J.; Barin, A. S.; Anal. Methods 2018, 30, 3770.

11. http://www.anvisa.gov.br/datavisa/fila_bula/index.asp, accessed in November 2018.

12. Mahmoud, W. M. M.; Kümmerer, K.; Chemosphere 2012, 88, 1170.

13. Nogueira, R.; Wollinger, W.; Silva, T. E.; Oliveira, L. M.; Rego, E. C. P.; Barin, J. S.; Laporta, L. V.; Mesko, M. F.; Bittencourt, C. F.; Rodrigues, J. M.; Cunha, V. S.; Moreira, G. F.; Braz. J. Pharm. Sci. 2011, 47, 351.

14. Gałuszka, A.; Migaszewski, Z.; Namieśnik, J.; TrAC, Trends Anal. Chem. 2013, 50, 78.

15. Melchert, W. R.; Reis, F. B.; Rocha, F. R. P.; Anal. Chim. Acta 2012, 714,8 .

Submitted: August 5, 2018

Published online: December 11, 2018 